\title{
Pegylation of Nanoliposomal Paclitaxel Enhances its Efficacy in Breast Cancer
}

\author{
Maedeh Koohi Moftakhari Esfahani ${ }^{1}$, Seyed Ebrahim Alavi ${ }^{1}$, Azim Akbarzadeh $^{2}$, \\ Soheil Ghassemi ${ }^{2}$, Zahra Saffari ${ }^{2}$, Maryam Farahnak ${ }^{2}$ and Mohsen Chiani ${ }^{2 *}$ \\ ${ }^{1}$ Engineering Department, Science and Research Branch, Islamic Azad University, ${ }^{2}$ Pilot Nanobiotechnology Department, \\ Pasteur Institute of Iran, Tehran, Iran
}

*For correspondence: Email: chiani110@yahoo.com; Tel: +98 21669688 56; Fax: +98 2166465132

\begin{abstract}
Purpose: To encapsulate paclitaxel into nanoliposomes, followed by pegylatation, in order to improve its therapeutic index and reduce side effects in breast cancer.

Methods: In order to prepare nanoliposomal paclitaxel, varying ratios of phosphatidylcholine, cholesterol and paclitaxel were mixed and the formulations pegylated with poly-ethylene glycol 2000 (PEG 2000) to enhance stability, efficiency, as well as solubility. The mean diameter of nanoliposomal paclitaxel and pegylated nanoliposomal paclitaxel were measured by Zeta sizer device and release of paclitaxel from both formulations was determined within $28 \mathrm{~h}$ by dialysis method. The cytotoxicity of nanoliposomal and pegylated nanoliposomal paclitaxel was evaluated using 3-(4,5-dimethylthiazol-2-yl)2,5-diphenyltetrazolium bromide (MTT) assay.

Results: The mean diameter of nanoliposomal paclitaxel and pegylated nanoliposomal paclitaxel was 421.4 and $369.1 \mathrm{~nm}$, respectively, while encapsulation efficiency was $91.3 \pm 5.7$ and $95.2 \pm 6.3 \%$, respectively. Paclitaxel released from both formulations in $28 \mathrm{~h}$ was 5.53 and $5.02 \%$, respectively. The cytotoxicity of pegylated nanoliposomal paclitaxel was significantly $(p<0.05)$ greater than that of nanoliposomal paclitaxel (their $I C_{50}=79.8 \pm 2.9$ and $86.25 \pm 3.4 \mu \mathrm{g} / \mathrm{ml}$, respectively).

Conclusion: The release pattern and cytotoxicity of pegylated nanoliposomal paclitaxel show that the formulation is superior to nanoliposomal paclitaxel. Furthermore, the mean particle size of pegylated nanoliposome is smaller than that of the non-pegylated preparation.
\end{abstract}

Keywords: Paclitaxel, Nanoliposome, Breast cancer, Pegylation, Drug delivery, Cytotoxicity

Tropical Journal of Pharmaceutical Research is indexed by Science Citation Index (SciSearch), Scopus, International Pharmaceutical Abstract, Chemical Abstracts, Embase, Index Copernicus, EBSCO, African Index Medicus, JournalSeek, Journal Citation Reports/Science Edition, Directory of Open Access Journals (DOAJ), African Journal Online, Bioline International, Open-J-Gate and Pharmacy Abstracts

\section{INTRODUCTION}

Cancer is now one of the most common diseases around the world. In 2007, the number of sufferers were about 11.3 million and it was predicted that this number would rise to 15.5 million by 2030 [1]. Breast cancer is commonest in women [2] and one million cases are annually diagnosed in the world [3]. It is the second commonest cause of cancer death in women [4].
Breast cancer ranks first among cancers in Iranian women [5]. The incidence of this disease in Iranian women is a decade younger than that of developed countries and the mean age of sufferers is reported 47.1 to 48.8 years [6]. Chemotherapy, radiation therapy and surgery are the ways by which breast cancer could be treated [1]. Paclitaxel is one of the drugs used to treat breast cancer. It belongs to taxanes family of anti-tumor activity $[7,8]$. This anti-cancer agent is used to treat ovarian, breast, head and neck, 
and non-small cell lung cancers $[8,9,10]$. Despite its therapeutic effect, paclitaxel chemotherapy has two major problems: its administration route and resistance [11].

In order to improve paclitaxel therapeutic effect, liposomal nanodrug delivery technology could be applied. Nanotechnology advancement creates new hope for cancer patients. Injectable nanosized carriers are used to cross biological barriers, protect the drug and release optimal dosage of the drug [12]. Liposome is one of these carriers.

Liposomes are colloidal, vesicular structures composed of one or more lipid bilayers surrounding an equal number of aqueous compartments $[13,14]$. They protect the drug against degradation and the patient against side effects of the drug [12]. This study aims to improve therapeutic effects and reduce side effects of paclitaxel.

\section{EXPERIMENTAL}

Phosphatidylcholine, cholesterol, polyethylene glycol 2000 (PEG 2000), paclitaxel and MTT solution $(0.5 \mathrm{mg} / \mathrm{ml})$ were purchased from Sigma Chemical Co., USA. Ethanol and isopropanol and RPMI 1640 medium were purchased from Merck Co. and Invitrogen Co., respectively. Also, breast cancer cell line (MCF-7) was obtained from Pasteur Institute, Iran.

\section{Nanoliposomal preparation, drug loading and pegylation}

A mixture of phosphatidylcholine and cholesterol $(1: 15 \mathrm{v} / \mathrm{v})$ was dissolved in $120 \mathrm{ml}$ of ethanol 98 $\%$ (300 rpm and room temperature) resulting in a clear and yellow suspension. Then, $13 \mathrm{mg}$ of paclitaxel was added and mixed by magnetic stirrer (300 rpm, $30 \mathrm{~min}$ and room temperature). Afterward, the solvent phase was evaporated by rotary evaporator (Heidolph Co., Germany) and the resultant film thin layer was dissolved in 13 $\mathrm{ml}$ of physiologic serum. Also in addition to above materials, PEG 2000 was added to prepare pegylated nanoliposomal paclitaxel. Then for $5 \mathrm{~min}$, both formulations was sonicated (Bandelin Sonorex Digitec, $60 \mathrm{~Hz}$ ).

\section{Size measurement of nanoliposomes}

The mean diameter of nanoliposomes was measured by Zeta sizer device (Malvern Instruments Ltd).

\section{Encapsulation efficiency}

Some of the obtained solution was centrifuged (13000 rpm, $30 \mathrm{~min}$ and $4{ }^{\circ} \mathrm{C}$ ) to study the amount of drug encapsulated. Afterward, the light absorbance of supernatant of each formulation was measured at $227 \mathrm{~nm}$ by a spectrophotometer (UV-1601 PC, Shimadzu Co.). Thereafter, encapsulation efficiency was calculated as in Eq 1:

$\mathrm{EE}(\%)=(\mathrm{ED} / \mathrm{TD}) 100$

where $E E$ is encapsulation efficiency, $E D$ is $t$ amount of encapsulated drug and TD is the total drug.

Different concentrations of paclitaxel were prepared and absorbance was measured at 227 $\mathrm{nm}$ spectrophotometerically to plot the standard curve.

\section{In vitro release study}

The study of the pattern of drug release from liposomes was carried out while $1 \mathrm{mg}$ of each liposomal and pegylated liposomal drug formulation was poured in a dialysis bag in 100 $\mathrm{ml}$ of phosphate buffered saline (PBS) of $\mathrm{pH} 7.4$ and placed on a magnetic stirrer $(24 \mathrm{~h}$ and 37 $\left.{ }^{\circ} \mathrm{C}\right)$. Then, the drug released in PBS was measured at $227 \mathrm{~nm}$ spectrophotometrically and the percentage of drug release was obtained using the drug standard curve.

\section{Cytotoxicity assay}

After MCF-7 cell culture, $100 \mu$ of suspension containing 10000 cells was decanted in 96 well plates and incubated $\left(5 \% \mathrm{CO}_{2}\right.$ and $\left.37^{\circ} \mathrm{C}\right)$. The supernatant of cells was removed after 24 hours and different concentrations of nanoliposomal paclitaxel and pegylated nanoliposomal paclitaxel and their controls were poured on cells and then incubated for $24 \mathrm{~h}$. Afterward, the supernatant was removed and $100 \mu$ of MTT solution $(0.5 \mathrm{mg} / \mathrm{ml})$ was added and after $3 \mathrm{~h}$ incubation, purple coloration was observed (as to the formation of formazane) and that mixture was then dissolved in live cells in $100 \mu \mathrm{l}$ of isopropanol. Light absorbance was measured at $570 \mathrm{~nm}$ by the Power Eave XS spectrophotometer and $\mathrm{IC}_{50}$ was calculated using using Pharm PCS (Pharmacologic Calculation System) statistical package (Springer Verlag, USA).

\section{Statistical analysis}

The results are expressed as mean \pm standard deviation ( $S D, n=3$ ). The data were statistically 
analyzed by one-way analysis of variance using IBM Statistics SPSS software version 19, and statistical significance was set at $p<0.05$.

\section{RESULTS}

\section{Particle size of nanoliposomes}

The mean diameter of liposomes for nanoliposomal and pegylated nanoliposomal drug was 421.4 and 369.1, respectively.

\section{Encapsulation efficiency}

Encapsulation efficiency (EE, \%) of each sample was calculated with respect to standard curve. Considering the formulation of encapsulation efficiency, the amount of non-encapsulated drug obtained was such that the percentage of encapsulated drug was calculated by subtracting it from the initial amount of drug. The encapsulation efficiency for nanoliposomal paclitaxel and pegylated nanoliposomal paclitaxel obtained were $91.3 \pm 5.7$ and $95.2 \pm$ $6.3 \%$, respectively.

\section{In vitro drug release}

Using the standard curve of paclitaxel, the amount of released paclitaxel of both nanoliposomal and pegylated nanoliposomal formulations in PBS were 5.53 and $5.02 \%$, respectively $30 \mathrm{~min}, 1,2,3,24$ and $28 \mathrm{~h}$ time intervals (Fig 1).

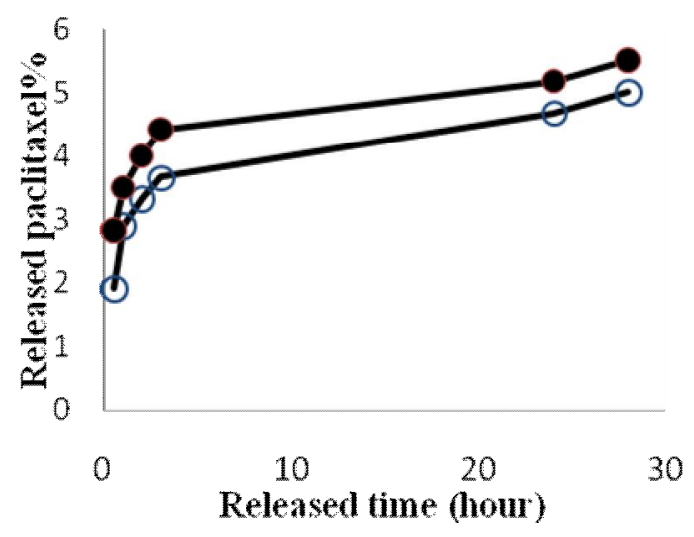

Figure 1: A comparison between drug releasing of liposomal paclitaxel $(\bullet)$ and pegylated liposomal paclitaxel $(\mathrm{O})$

\section{Cytotoxicity}

Drug toxicity was examined at different concentrations based on MTT technique. The $\mathrm{IC}_{50}$ for pegylated nanoliposomal paclitaxel, nanoliposomal paclitaxel and standard paclitaxel were $79.8 \pm 2.9,86.25 \pm 3.4$ and $142 \pm 6.6 \mu \mathrm{g} / \mathrm{ml}$, respectively.

\section{DISCUSSION}

Drug delivery is one of the main challenges of pharmaceutical biotechnology [15]. It has been shown that nano carriers manage to deliver drugs to targeted cells. Liposome is one of the lipid nano carriers. Liposomes protect a drug against degradation whereas they protect patient against side effects of encapsulated drug [12].

Ram et al targeted doxorubicin encapsulated in liposome to cells containing surface markers CD44. The results indicate the clear effect of doxorubicin encapsulated in liposome against CD44 expressing cells [16]. In a research conducted by Sharam et al, liposome was investigated as a drug delivery system in breast cancer which its results illustrated the important effect of drugs encapsulated in liposomes labeled by antibodies against tumor cell antigens in diagnosis and treatment of breast cancer [17]. Giota et al examined doxorubicin encapsulated in liposome in cyclophosphamide as the first line in treatment of breast cancer. The findings obtained indicate again the increment of toxicity of doxorubicin encapsulated in liposome in comparison with standard drug [18].

The present study investigated the cytotoxicity effect of both nanoliposomal and pegylated nanoliposomal paclitaxel formulations on MCF-7.

The mean diameter of both nanoliposomal and pegylated nanoliposomal formulations was measured by Zeta sizer device after their fabrication. Results showed that the particle size of pegylated nanoliposomal formulation was significantly less than that of nanoliposomal formulation. PEG penetrates to liposomal layers and presses them together as it has a hydrophilic nature and high permeability, seemingly the cause of the smaller particle size of pegylated nanoliposomal formulation.

The examination of paclitaxel encapsulation showed that the encapsulation of nanoliposomal drug was $3.9 \%$ less than that of pegylated nanoliposomal drug. As PEG envelopes the nanoliposome containing paclitaxel, it apparently leads to slower drug leakage and more drug stability. Also, PEG makes insoluble drugs, such as paclitaxel, soluble which results in encapsulation increment. 
The cytotoxic effect of both nanoliposomal and pegylated nanoliposomal paclitaxel formulations was perused by MTT assay in which both formulations without any drug did not represent any cytotoxicity effect on MCF-7. The results indicated that the least $I_{50}$ belonged to pegylated nanoliposomal paclitaxel and the $\mathrm{IC}_{50}$ of nanoliposomal paclitaxel was less than that of standard drug. This phenomenon seems to stem from the effect of PEG on more stability and slower drug releasing of pegylated nanoliposomal formulation. Also, PEG increases drug solubility and its collision with the targeted cell.

\section{CONCLUSION}

Nanoliposomal and pegylated nanoliposomal formulation of paclitaxel can be prepared by reverse phase evaporation method. The results indicate that most of the drug is encapsulated into the carrier and cytotoxicity was lower than that of the standard drug. Furthermore, in vitro drug release was slower from the developed formulations.

\section{ACKNOWLEDGEMENT}

The authors are grateful to all the staff of Pilot Nanobiotechnology Department, Pasteur Institute of Iran for their assistance and guidance.

\section{REFERENCES}

1. Guo J, Bourre L, Soden DM, O'Sullivan GC, O'Driscoll C. Can non-viral technologies knockdown the barriers to siRNA delivery and achieve the next generation of cancer therapeutics Biotechnol Adv. 2011; 29(4): 402-417.

2. Warner E. Clinical practice. Breast-cancer screening. $N$ Engl J Med. 2011; 365(11): 1025-1032.

3. Anders CK, Carey LA. Biology, metastatic patterns, and treatment of patients with triple-negative breast cancer. Clin Breast Cancer. 2009; 9 Suppl 2: S7381.

4. American Cancer Society, Cancer Facts \& Figures, America Cancer Society, Atlanta, GA, USA, 2011.
5. Sadjadi A, Nouraie M, Mohagheghi MA, Mousavi-Jarrahi A, Malekezadeh R, Parkin DM. Cancer occurrence in Iran in 2002, an international perspective. Asian Pac J Cancer Prev. 2005; 6(3): 359-363.

6. Harirchi I, Karbakhsh M, Kashefi A, Momtahen AJ. Breast cancer in Iran: results of a multi-center study. Asian Pac J Cancer Prev. 2004; 5(1): 24-27.

7. Abal M, Andreu JM, Barasoain I. Taxanes: microtubule and centrosome targets, and cell cycle dependent mechanisms of action. Curr Cancer Drug Targets. 2003; 3(3): 193-203.

8. Rowinsky EK, Onetto N, Canetta RM, Arbuck SG. Taxol: the first of the taxanes, an important new class of antitumor agents. Semin Oncol. 1992; 19(6): 646662.

9. Greco FA. Paclitaxel-based combination chemotherapy in advanced non-small cell lung cancer. Lung Cancer. 2001; 34(Suppl 4): S53-S56.

10. Rowinsky EK, Donehower RC. Paclitaxel (taxol). N Engl J Med. 1995 Apr 13; 332(15): 1004-1014.

11. Dorr RT. Pharmacology and toxicology of Cremophor EL diluent. Ann Pharmacother. 1994; 28(5 Suppl): S11S14.

12. Costantino L, Boraschi D. Is there a clinical future for polymeric nanoparticles as brain-targeting drug delivery agents? Drug Discov Today. 2012; 17(7-8): 367-378.

13. Maurer N, Fenske DB, Cullis PR. Developments in liposomal drug delivery systems. Expert Opin Biol Ther. 2001; 1(6): 923-947.

14. Latif N, Bachhawat BM. Liposomes in immunology. J. Biosci., Vol. 6, Number 4, 1984; pp. 491- 502.

15. Georgens C, Weyermann J, Zimmer A. Recombinant virus like particles as drug delivery system. Curr Pharm Biotechnol. 2005; 6(1): 49-55.

16. Eliaz RE, Szoka FC Jr. Liposome-encapsulated doxorubicin targeted to CD44: a strategy to kill CD44overexpressing tumor cells. Cancer Res. 2001; 15; 61(6): 2592-2601.

17. Sharma G, Anabousi S, Ehrhardt C, Ravi Kumar MN. Liposomes as targeted drug delivery systems in the treatment of breast cancer. J Drug Target. 2006; 14(5): 301-310.

18. Giotta F, Lorusso V, Maiello E, Filippelli G, Valerio MR, Caruso M, Verderame F, Latorre A, Colucci $G$. Liposomal-encapsulated doxorubicin plus cyclophosphamide as first-line therapy in metastatic breast cancer: a phase II multicentric study. Ann Oncol. 2007; 18(Suppl 6): vi66-vi69. 